Document downloaded from:

http://hdl.handle.net/10251/176368

This paper must be cited as:

Vidal-Ferràndiz, A.; Carreño, A.; Ginestar Peiro, D.; Demazière, C.; Verdú Martín, GJ. (2020). Neutronic Simulation of Fuel Assembly Vibrations in a Nuclear Reactor. Nuclear Science and Engineering. 194(11):1067-1078. https://doi.org/10.1080/00295639.2020.1756617

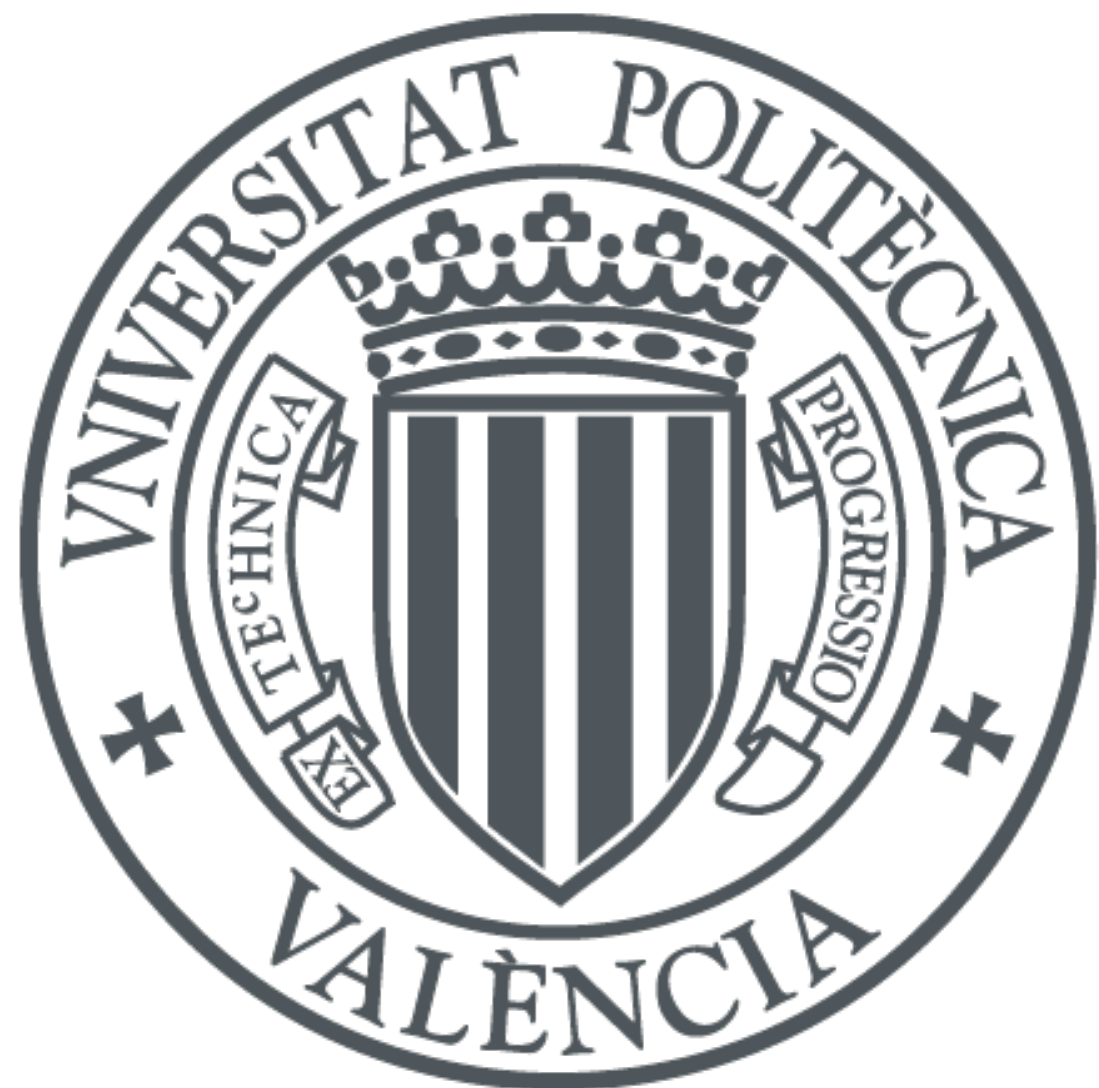

The final publication is available at

https://doi.org/10.1080/00295639.2020.1756617

Copyright Taylor \& Francis

Additional Information

This is an Accepted Manuscript of an article published by Taylor \& Francis in Nuclear Science and Engineering on 2020, available online:

http://www.tandfonline.com/10.1080/00295639.2020.1756617 


\title{
Neutronic Simulation of Fuel Assembly Vibrations in a Nuclear Reactor
}

\author{
A. Vidal-Ferràndiz, ${ }^{*}$ a A. Carreño, ${ }^{a}$ D. Ginestar, ${ }^{\mathrm{a}}$ C. Demazière, ${ }^{\mathrm{b}}$ and G. Verdú ${ }^{\mathrm{a}}$ \\ ${ }^{a}$ Universitat Politècnica de València, \\ Cami de Vera s/n, 46022, València, Spain \\ ${ }^{b}$ Chalmers University of Technology, \\ Department of Physics, Division of Subatomic and Plasma Physics, \\ SE-412 96 Gothenburg, Sweden \\ *Email: anvifer2@upv.es
}

Number of pages: 22

Number of tables: 1

Number of figures: 12 


\begin{abstract}
The mechanical vibrations of core internals as fuel assemblies cause oscillations in the neutron flux that require, in some circumstances, to operate nuclear power plants at a reduced power level. This work simulates and analyses the changes of the neutron flux throughout a nuclear core due to the oscillation of a single fuel assembly without considering thermal-hydraulic feedback. The amplitude of the fuel assembly vibration is bounded to a few millimeters and this implies the use of fine meshes and accurate numerical solvers due to the different scales of the problem. The results of the simulations show a main oscillation of the neutron flux with the same frequency as the fuel assembly vibration along with other harmonics at multiples of the vibration frequency much smaller in amplitude. Also, this work compares time-domain analysis and frequency-domain analysis of the mechanical vibrations. Numerical results show a close match between these two approaches for the fundamental frequency.
\end{abstract}

Keywords - Neutron Noise, Fuel Assembly Vibrations, Neutron Diffusion Approximation, Timedomain modeling, Frequency-domain modeling 


\section{INTRODUCTION}

In the area of core monitoring, detection of reactor perturbations gives the possibility to take proper actions before such problems lead to safety concerns or impact plant availability. The CORTEX project [1], aims at developing an innovative core monitoring technique that allows detecting anomalies in nuclear reactors, such as excessive vibrations of core internals, flow blockage, coolant inlet perturbations... The technique is based on using the fluctuations of the neutron flux around its steady state value, known as neutron noise, recorded by in-core and ex-core detectors. The main benefit of this method is that it can be applied on-line without disrupting plant operation. Furthermore, the method is non-intrusive, i.e. no additional perturbation needs to be introduced.

A correlation between the stiffness of fuel assemblies (FA) and neutron noise levels was experimentally demonstrated [2], indicating the direct effect of FA vibrations onto the neutron noise. This paper asses the capability of time-domain and frequency-domain simulations to study the spatial dependence of the neutron noise caused by FA vibrations. Also, it evaluates the magnitude and spatial shape of the neutron noise when a single FA is vibrating. Reactor core instrumentation is capable of detecting a small periodic variation at a specific frequency and it can be differentiated from the random noise in the detectors signals (white noise) $[3,4]$. The detection of neutron noise nevertheless requires a specific signal processing, both using hardware and software: removal of the mean value, amplification, anti-aliasing filters, and analog-to-digital conversion. The analysis of neutron noise is then most easily and most efficiently performed in the frequency-domain, where peaks in the spectra can be identified and subsequently analyzed. FA vibrations has received some particular interest in the CORTEX project, with the COLIBRI experiments in the CROCUS reactor [5]. These experiments mimic fuel assembly vibrations by displacing fuel rods in an oscillatory manner. These experiments also demonstrate that such vibrations induce a neutron noise measurable by the core instrumentation.

The present contribution simulates the movement of a FA as a sinusoidal displacement of the boundary between adjacent homogenized regions. Other studies considered FA vibrations as random modification of the size of the water gaps which surrounds the FA of interest [6]. Timedomain and frequency-domain comparisons of different perturbations have been reported lately in $[7,8,9]$.

FA vibrations have been studied from a mechanical point of view in [10] and [3]. Natural 
frequencies range from $0.8 \mathrm{~Hz}$ to $24.5 \mathrm{~Hz}$ depending on the idealized form of bearing and their building materials. The amplitude of the vibrations ranges can reach up to $1 \mathrm{~mm}$. However, there can be amplitudes greater than $1 \mathrm{~mm}$ in case of synchronous motions. This work is based on a single FA vibration of $1 \mathrm{~Hz}$ and $1 \mathrm{~mm}$ of amplitude as a typical example.

This paper is an extension of [11] and is organized as follows. First, the time-domain analysis is explained in Section II. Section III describes the frequency-domain analysis and the usual approximation to model a mechanical FA vibration. Then, numerical results for a two-dimensional model are given in Section IV to compare the proposed methodologies. Finally, Section V summarizes the main conclusions of the work.

\section{TIME-DOMAIN ANALYSIS}

The neutron transport equation for nuclear reactor applications is usually approximated by the neutron diffusion equation. For a given transient, the balance of neutrons inside a nuclear reactor core can be modeled using the time-dependent neutron diffusion equation in the two energy groups approximation without up-scattering, which is of the form

$$
\begin{aligned}
{\left[\mathrm{v}^{-1}\right] \frac{\partial \Phi}{\partial t}+\mathcal{L} \Phi } & =\left(1-\beta_{\mathrm{eff}}\right) \mathcal{M} \Phi+\sum_{k=1}^{K} \lambda_{k} \chi \mathcal{C}_{k}, \\
\frac{\partial \mathcal{C}_{k}}{\partial t} & =\beta_{k}\left[\nu \Sigma_{f 1} \nu \Sigma_{f 2}\right] \Phi-\lambda_{k} \mathcal{C}_{k}, \quad k=1, \ldots, K,
\end{aligned}
$$

where $K$ is the number of delayed neutron precursors groups considered and the following matrices have been defined

$$
\begin{aligned}
& \mathcal{L}=\left(\begin{array}{cc}
-\vec{\nabla} \cdot\left(D_{1} \vec{\nabla}\right)+\Sigma_{a 1}+\Sigma_{12} & 0 \\
-\Sigma_{12} & -\vec{\nabla} \cdot\left(D_{2} \vec{\nabla}\right)+\Sigma_{a 2}
\end{array}\right), \quad\left[\mathrm{v}^{-1}\right]=\left(\begin{array}{cc}
\frac{1}{\mathrm{v}_{1}} & 0 \\
0 & \frac{1}{\mathrm{v}_{2}}
\end{array}\right), \\
& \mathcal{M}=\left(\begin{array}{cc}
\nu \Sigma_{f 1} & \nu \Sigma_{f 2} \\
0 & 0
\end{array}\right), \quad \Phi=\left(\begin{array}{l}
\phi_{1} \\
\phi_{2}
\end{array}\right), \quad \chi=\left(\begin{array}{l}
1 \\
0
\end{array}\right) \text {, }
\end{aligned}
$$

where $\phi_{1}$ and $\phi_{2}$ are the fast and the thermal neutron fluxes, respectively. Other quantities have their usual notation, see for example [12]. Although a transport-based solution without spatial homogenization would be required to properly catch the effect of local noise sources, some studies 
revealed that a diffusion-based solution correctly represents a transport-based solution a few mean free paths from the noise source and from strong heterogeneities [13, 14].

In this work, a high order finite element method (FEM) has been used for the spatial discretization of the neutron diffusion equation, as the one presented in [15] and [16].

Once the spatial discretization has been selected, a discrete version of the time-dependent neutron diffusion equation is solved. Since the system of ordinary differential equations resulting from the discretization of the neutron diffusion equations is, in general, stiff, implicit methods are necessary. Particularly, a first order backward method is used [17]. This method leads to a large system of linear equations that needs to be solved for each time step. The semi-discrete two energy groups time-dependent neutron diffusion equation together with the neutron precursors concentration equations are of the form

$$
\begin{aligned}
{\left[\tilde{\mathrm{v}}^{-1}\right] \frac{d \Phi}{d t}+L \Phi } & =\left(1-\beta_{\mathrm{eff}}\right) M \Phi+\sum_{k=1}^{K} \lambda_{k} X C_{k}, \\
P \frac{d C_{k}}{d t} & =\beta_{k}\left(M_{11} M_{12}\right) \Phi-\lambda_{k} P C_{k}, \quad k=1, \ldots, K,
\end{aligned}
$$

where $L$ and $M$ are the matrices obtained from the spatial discretization of operators $\mathcal{L}$ and $\mathcal{M} . \Phi$ and $C_{k}$ are the vector of coefficients of the neutron flux and the precursors concentration in terms of the polynomials used in the FEM. $M_{11}, M_{12}$ are the non-zero block matrices of the matrix $M$. The matrices $X$ and $\left[\tilde{\mathrm{v}}^{-1}\right]$ are defined as

$$
X=\left(\begin{array}{c}
P \\
0
\end{array}\right), \quad\left[\tilde{\mathrm{v}}^{-1}\right]=\left(\begin{array}{cc}
P \mathrm{v}_{1}^{-1} & 0 \\
0 & P \mathrm{v}_{2}^{-1}
\end{array}\right)
$$

where the matrix $P$ is the mass matrix of the spatial discretization, that appears because the polynomial basis used in the spatial discretization is not orthonormal.

The neutron precursors equation (4) can be discretized in time using an explicit scheme as

$$
P C_{k}^{n+1}=P C_{k}^{n} e^{-\lambda_{k} \Delta t}+\frac{\beta_{k}}{\lambda_{k}}\left(1-e^{-\lambda_{k} \Delta t}\right)\left(M_{11}^{n+1} M_{12}^{n+1}\right) \Phi^{n+1} .
$$

In the same way, Euler's backward method is used in equation (3), using equation (5), yielding 
to a set of linear equations,

$$
T^{n+1} \Phi^{n+1}=R^{n} \Phi^{n}+\sum_{k=1}^{K} \lambda_{k} e^{-\lambda_{k} \Delta t} X C_{k}^{n},
$$

where the matrices are defined as,

$$
\begin{aligned}
T^{n+1} & =\frac{1}{\Delta t}\left[\tilde{\mathrm{v}}^{-1}\right]+L^{n+1}-\hat{a} M^{n+1}, \\
R^{n} & =\frac{1}{\Delta t}\left[\tilde{\mathrm{v}}^{-1}\right]=\frac{1}{\Delta t}\left(\begin{array}{cc}
P \mathrm{v}_{1}^{-1} & 0 \\
0 & P \mathrm{v}_{2}^{-1}
\end{array}\right),
\end{aligned}
$$

and the coefficient $\hat{a}$ is

$$
\hat{a}=1-\beta_{\mathrm{eff}}+\sum_{k=1}^{K} \beta_{k}\left(1-e^{-\lambda_{k} \Delta t}\right)
$$

where $\beta_{\text {eff }}=\sum_{k=1}^{K} \beta_{k}$. This system of equations has to be solved for each time step. The preconditioned BICGSTAB method [18] has been chosen to solve these systems and the preconditioner used has been the incomplete LU preconditioner together with a reordering of the matrix to decrease the fill-in. This system of equations is large and sparse and has to be solved for each new time step with a high accuracy, tol $=10^{-12}$.

The finite element method used in this work has been implemented using the open source finite elements library deal.II [19]. To solve the resulting algebraic eigenvalue and linear problems the libraries PETSc [20] and SLEPc [21] are used. The code developed is called FEMFFUSION.

Finally, to compare the results obtained from the time-domain analysis with the ones obtained with the frequency-domain methodology we define the neutron noise as

$$
\delta \phi(\mathbf{r}, t)=\phi(\mathbf{r}, t)-\phi(\mathbf{r}, 0)
$$

Then, we can apply a Fourier transform to the neutron noise in the time-domain,

$$
\delta \phi(\mathbf{r}, \omega)=\mathcal{F}[\delta \phi(\mathbf{r}, t)]=\int_{-\infty}^{\infty} \exp (-i \omega t) \delta \phi(\mathbf{r}, \omega) \mathrm{d} t .
$$

This transform is calculated with the Fast Fourier Transform algorithm (FFT) over the timedependent results using a sampling time of $10^{-2} \mathrm{~s}$ and scaled accordingly to be able to compare 
the discrete Fourier transform results with the continuous Fourier transform given by equation (8).

\section{FREQUENCY-DOMAIN ANALYSIS}

The neutron diffusion equation in the frequency-domain has been already successfully used to study other neutron noise sources $[22,23,24]$. One usual tool to solve the two-group diffusion equation in the frequency-domain in the first-order neutron noise approximation is the CORE SIM code [25]. This tool has been already validated against many (semi-)analytical solutions [26] and other solutions.

The first-order neutron noise theory is based on separating every time-dependent term, expressed as $U(\mathbf{r}, t)$, into their mean value, $U_{0}$, and their fluctuation around their mean value, $\delta U_{0}$ as

$$
U(\mathbf{r}, t)=U_{0}(\mathbf{r})+\delta U(\mathbf{r}, t) .
$$

The fluctuations are assumed to be small compared to the mean values. This allows to neglect second-order term $(\delta U(\mathbf{r}, t) \times \delta U(\mathbf{r}, t)) \approx 0$. Also, the fluctuations of the diffusion coefficients are neglected and $\delta D_{g}=0$ is assumed. Then, a Fourier transform is applied to the neutron diffusion equation. Thus, the first-order neutron noise equation can be written as [27],

$$
\left(\vec{\nabla} D \vec{\nabla}+\Sigma_{\mathrm{dyn}}\right)\left(\begin{array}{c}
\delta \phi_{1} \\
\delta \phi_{2}
\end{array}\right)=\delta \Sigma_{r} \phi_{r}+\phi_{a}\left(\begin{array}{c}
\delta \Sigma_{a 1} \\
\delta \Sigma_{a 2}
\end{array}\right)+\phi_{f}\left(\begin{array}{c}
\delta \Sigma_{f 1} \\
\delta \Sigma_{f 2}
\end{array}\right)
$$

where

$$
\begin{aligned}
& D=\left(\begin{array}{cc}
D_{1} & 0 \\
0 & D_{2}
\end{array}\right), \quad \Sigma_{\mathrm{dyn}}=\left(\begin{array}{cc}
-\Sigma_{1} & \nu \Sigma_{f 2}\left(1-\frac{i \omega \beta_{\text {eff }}}{i \omega+\lambda_{\text {eff }}}\right) \\
\Sigma_{12} & \left(\Sigma_{a 2}+\frac{i \omega}{\mathrm{v}_{2}}\right)
\end{array}\right), \quad \phi_{r}=\left(\begin{array}{c}
\phi_{1} \\
-\phi_{1}
\end{array}\right), \\
& \phi_{a}=\left(\begin{array}{cc}
\phi_{1} & 0 \\
0 & \phi_{2}
\end{array}\right), \quad \phi_{f}=\left(\begin{array}{cc}
-\phi_{1}\left(1-\frac{i \omega \beta_{\text {eff }}}{i \omega+\lambda_{\text {eff }}}\right) & -\phi_{2}\left(1-\frac{i \omega \beta_{\text {eff }}}{i \omega+\lambda_{\text {eff }}}\right) \\
0 & 0
\end{array}\right), \\
& \Sigma_{1}=\Sigma_{a}+\frac{i \omega}{\mathrm{v}_{1}}+\Sigma_{r}-\nu \Sigma_{f 1}\left(1-\frac{i \omega \beta_{\mathrm{eff}}}{i \omega+\lambda_{\text {eff }}}\right), \quad \lambda_{\text {eff }}=\frac{1}{\sum_{k=1}^{K} \frac{1}{\lambda_{k}}} .
\end{aligned}
$$

The neutron noise equation is a partial differential equation with complex coefficients that has to be solved after the static solution is obtained, as $\phi_{1}$ and $\phi_{2}$ represent the steady state fast and 
thermal neutron fluxes. The related static eigenvalue problem must be solved with the same spatial discretization as the frequency-domain neutron noise equation to get coherent results. CORE SIM uses a box finite difference scheme to solve the steady-sate and the frequency-domain neutron noise problems.

It must be noted that the frequency-domain approach must solve a linear system in the complex domain but this system is only solved once. On the other hand the time-domain approach must solve a linear system at each time step, resulting in a large amount of linear systems resolutions for a typical transient. Small time steps are usually used to solve the neutron diffusion equation when the $\left[\mathrm{v}^{-1}\right] \partial \Phi / \partial t$ term of equation (1) must be accurately represented. However, this term is important only when the neutron flux changes at least one decade over a ms. This is not the case of neutron noise analysis calculations. In this way, a time step of $\Delta t=10^{-2} \mathrm{~s}$ has been proven to give accurate results, although this work has used a time step of $\Delta t=10^{-4} \mathrm{~s}$.

\section{III.A. A vibrating FA in the frequency-domain}

There are different models to describe the vibration of a FA in the frequency domain. In this work, we used the $\epsilon / D$ model, in which the vibration is converted into a spatially localized cross-section perturbation [27].

Assuming a nodal representation of the system (i.e. each fuel assembly is replaced by homogenized regions), one oscillating FA can be modeled as two moving interfaces between homogeneous materials. For the sake of illustration, we consider hereafter two adjacent homogeneous regions and the corresponding possible displacement of the boundary between those regions, as shown in Figure 1. The cross section, $\Sigma_{\alpha}$, at the interface $x=b$ between two material regions can be described as

$$
\Sigma_{\alpha}(x)=(1-\mathcal{H}(x-b)) \Sigma_{\alpha}^{I}+\mathcal{H}(x-b) \Sigma_{\alpha}^{I I},
$$

where $\mathcal{H}$ is the unit step function, $\Sigma_{\alpha}^{I}$ and $\Sigma_{\alpha}^{I I}$ are the cross sections at region $I$ and $I I$, respectively.

A moving interface as $b(t)=b_{0}+A \sin \left(\omega_{p} t\right)$, results in:

$$
\Sigma_{\alpha}(x, t)=\left(1-\mathcal{H}\left(x-b_{0}-A \sin \left(\omega_{p} t\right)\right)\right) \Sigma_{\alpha}^{I}+\mathcal{H}\left(x-b_{0}-A \sin \left(\omega_{p} t\right)\right) \Sigma_{\alpha}^{I I}
$$

Using the first order Taylor expansion around $x-b_{0}$, the cross section perturbation can be expressed 


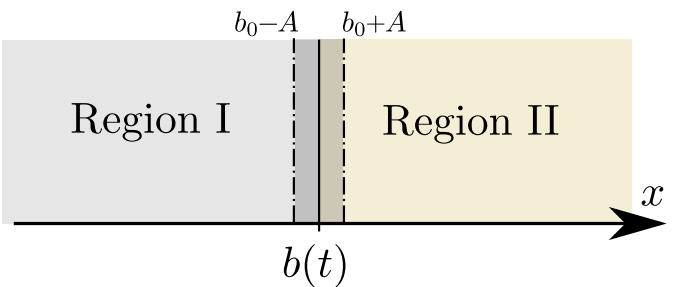

Fig. 1. Vibrating interface between two regions.

as

$$
\delta \Sigma_{\alpha}(x, t)=\left(\Sigma_{\alpha}^{I}-\Sigma_{\alpha}^{I I}\right) A \sin \left(\omega_{p} t\right) \delta\left(x-b_{0}\right)
$$

and, in the frequency-domain, the perturbation can be written as follows

$$
\delta \Sigma_{\alpha}(x, \omega)=-i \pi A\left(\Sigma_{\alpha}^{I}-\Sigma_{\alpha}^{I I}\right) \delta\left(x-b_{0}\right)\left(\delta\left(\omega-\omega_{p}\right)-\delta\left(\omega+\omega_{p}\right)\right)
$$

Since in numerical tools like CORE SIM the perturbation is introduced node-wise, one could assume that the perturbed region is $x \in\left[b_{0}-A, b_{0}+A\right]$ at angular frequency $\omega_{p}$ with an average value of $\delta \Sigma_{\alpha}=-\frac{1}{2} i \pi\left(\Sigma_{\alpha}^{I}-\Sigma_{\alpha}^{I I}\right)$ at the perturbed region. If the spatial mesh used does not match the perturbed region, the perturbation must be scaled accordingly. In [28] we can find the full analytical expression for the model of a vibrating FA in the frequency domain. This model highlights the fact that a monochromatic displacement introduces polychromatic perturbations of the homogenized cross-sections on which the boundary is moving. This is due to the fact that those homogenized regions are perturbed only during parts of the vibration period.

To test the accuracy of the first order approximation, a numerical fast Fourier transform of a time-dependent cross sections, $\Sigma_{\alpha}(x, t)$, is calculated. The numerical FFT was obtained for a one dimensional moving interface perturbation of $\Sigma_{\alpha}^{I}-\Sigma_{\alpha}^{I I}=1$ and $1 \mathrm{~Hz}$. Figure 2 shows the spectrum of the perturbation at different spatial points using the FFT. The amplitude of the cross section perturbation is maximum at $1 \mathrm{~Hz}$ and the amplitudes at other frequencies are smaller.

Figure 3 shows the absolute value of the amplitude of the perturbation, $\left|\delta \Sigma_{\alpha}\right|$, at $1 \mathrm{~Hz}$, $2 \mathrm{~Hz}, 3 \mathrm{~Hz}$ and $4 \mathrm{~Hz}$ for the numerical FFT and the first order approximation, in other words the amplitude of the perturbation at $\omega_{p}, 2 \omega_{p}, 3 \omega_{p}$ and $4 \omega_{p}$. Figure $3($ a) at $1 \mathrm{~Hz}$, shows that the main frequency of the cross section perturbation produced by a moving FA is the frequency 
of the oscillation. In this way, the first order approximation only uses the perturbation at this frequency to model the FA vibration. It must be noted that the first order approximation has the same perturbation integral as the FFT and it can be introduced using only one node. The higher harmonics corresponding to frequencies of $2 \mathrm{~Hz}, 3 \mathrm{~Hz}$ and $4 \mathrm{~Hz}$ usually are not taken into account in a frequency-domain code [25]. Here we study the contributions of those higher harmonics solving the time-dependent problem with FEMFFUSION. In this way, the time-domain approach does not decompose the perturbation and so does not imply any assumptions in the perturbation source. In [29] and [28] more results corresponding to the higher harmonics of the noise source are given. Moreover, different models of the noise induced from a mechanical vibration of a fuel assembly can be found.

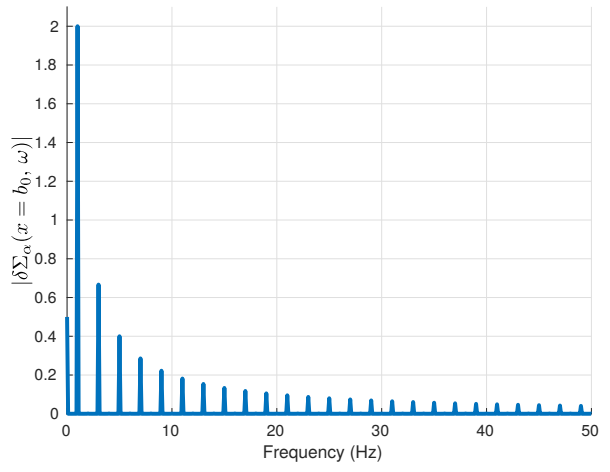

(a) At $b_{0}$.

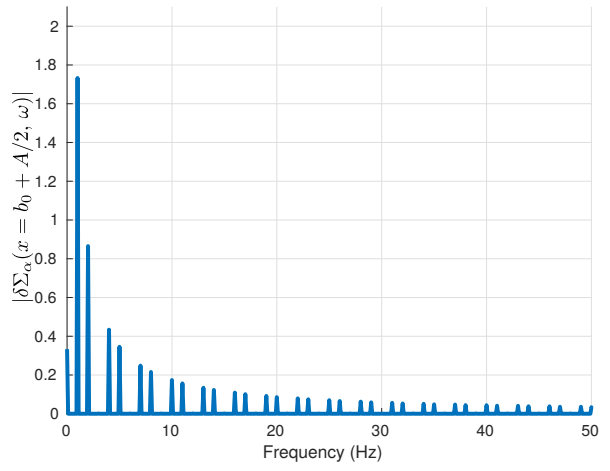

(c) At $b_{0}+A / 2$.

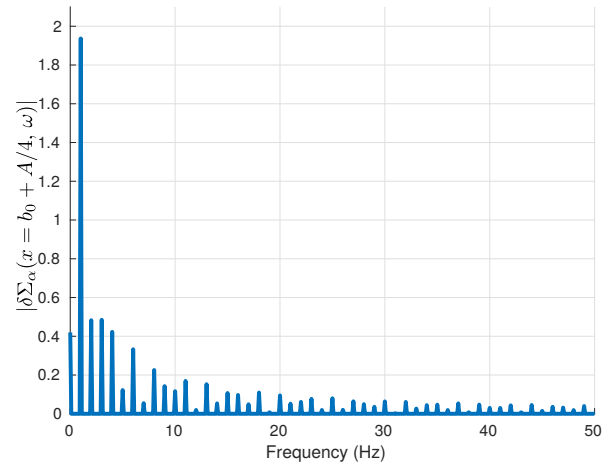

(b) At $b_{0}+A / 4$.

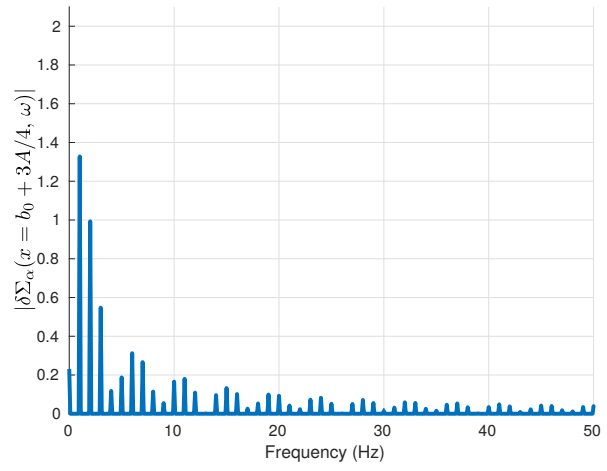

(d) At $b_{0}+3 A / 4$.

Fig. 2. Spectrum of the numerical FFT at different spatial points. 


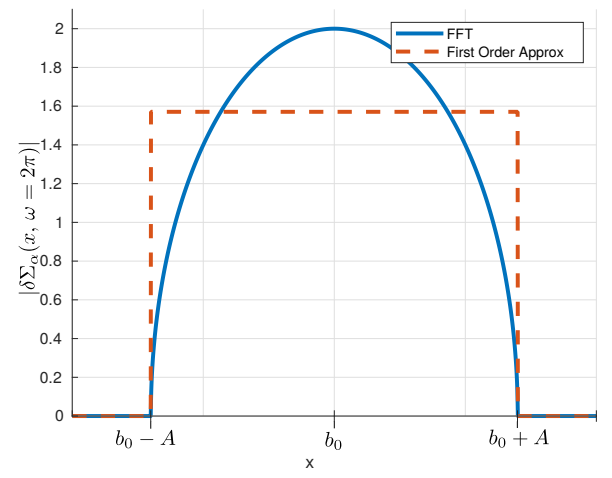

(a) At $1 \mathrm{~Hz}$

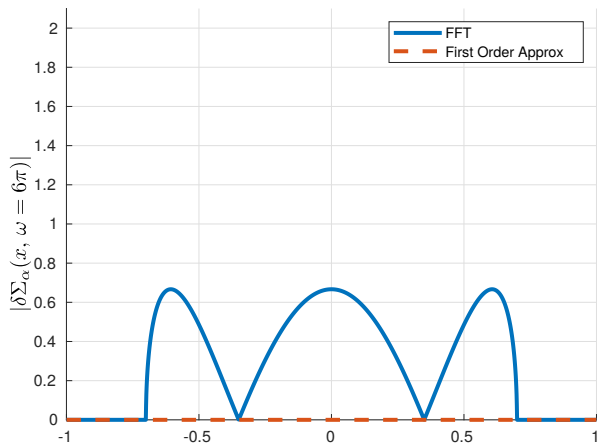

(c) At $3 \mathrm{~Hz}$

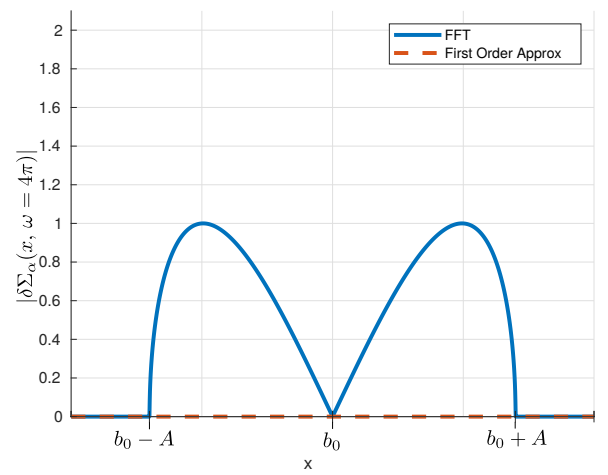

(b) At $2 \mathrm{~Hz}$

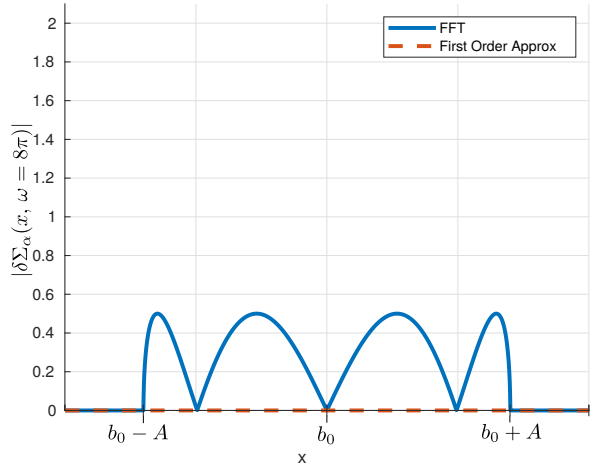

(d) At $4 \mathrm{~Hz}$.

Fig. 3. Cross Section perturbation amplitudes at different frequencies.

\section{NUMERICAL RESULTS}

The BIBLIS 2D benchmark is selected to compare the frequency and time-domain analysis for a vibrating FA. This is a classical two-group neutron diffusion problem taken as a benchmark for different numerical codes. The material and geometry definition of the benchmark can be found in [30]. The problem is made critical before starting the time-dependent calculation by dividing $\nu \Sigma_{f g}$ over $k_{\text {eff }}$.

The assembly in the position $(6,6)$ is selected to be oscillating along the $x$ direction as

$$
x_{i}(t)=x_{i 0}+A \sin \left(\omega_{p} t\right)
$$

where $x_{i}(t)$ represents each position of the vibrating assembly along time, originally placed at $x_{i 0}$. The selected amplitude of the movement is $1 \mathrm{~mm}$ and the frequency of the vibration is $1 \mathrm{~Hz}$, i.e. 
$\omega_{p}=2 \pi$. The perturbation for the time-domain calculation is inserted changing the cross sections at each time step, modeling the movement of the FA by volume-averaged cross sections. For the frequency-domain calculation, the perturbation is inserted following the first order approximation as explained in Section III.A. In this way, the perturbation is inserted cell wise in the adjacent cells next to the moving FA as shown in Figure 4. The value of the cross sections and the perturbed cross sections are given in Table I following the formula, $\delta \Sigma_{\alpha}=-i \frac{\pi}{2}\left(\Sigma_{\alpha}^{I}-\Sigma_{\alpha}^{I I}\right)$. Two perturbed regions are then defined: one on the left and one on the right of the moving boundary of the vibrating FA. These regions have the same cross section perturbation amplitude but a phase lag of $180^{\circ}$. Due to the different scales of the problem, a fine mesh needs to be used to accurately solve the system. In the time-domain analysis, a refined spatial mesh in the surroundings of the moving FA with 869 cells and cubic polynomials in the FEM are used. In the frequency-domain analysis, a uniform mesh of 4624 cells is employed. If these fine meshes are not used, the effect of the FA vibration could be overestimated [9].

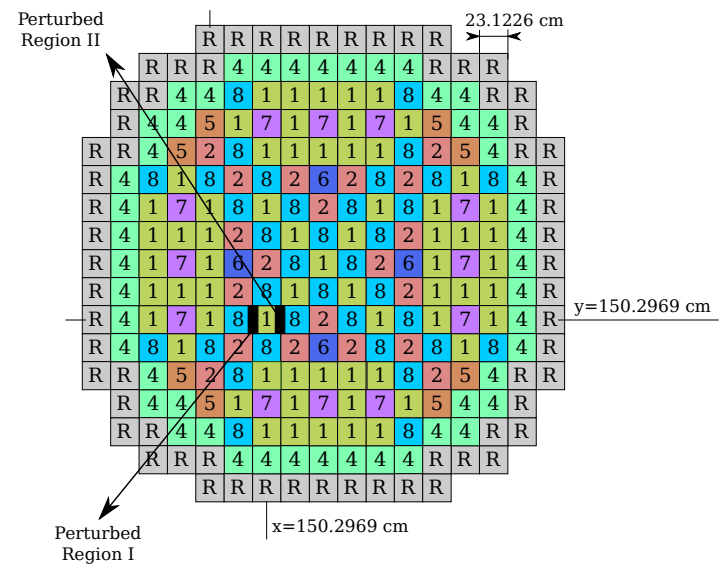

Fig. 4. Materials and perturbed regions of the 2D BIBLIS reactor.

Figure 5 shows the spatial distribution of the amplitude of the relative neutron noise in the frequency-domain calculated with the time-dependent code FEMFFUSION at $1 \mathrm{~Hz}$. In this figure, the relative neutron noise is represented in percentage of the steady-state neutron flux. It can be seen that the induced fast neutron noise has an influence on larger scales through the reactor compared to the thermal noise, due to the larger mean free path of fast neutrons. On the other hand, the thermal noise is localized in the surroundings of the oscillating FA. Two clear peaks can be observed in these Figures at the position of the moving interfaces where the cross sections 
TABLE I

Static and perturbation cross sections of the BIBLIS 2D reactor.

\begin{tabular}{|c|c|c|c|c|c|}
\hline Material & $\mathrm{g}$ & $\begin{array}{c}\boldsymbol{D}_{\boldsymbol{g}} \\
(\mathrm{cm})\end{array}$ & $\begin{array}{c}\boldsymbol{\Sigma}_{a \boldsymbol{g}} \\
(1 / \mathrm{cm})\end{array}$ & $\begin{array}{c}\boldsymbol{\nu} \boldsymbol{\Sigma}_{\boldsymbol{f g}} \\
(1 / \mathrm{cm})\end{array}$ & $\begin{array}{c}\boldsymbol{\Sigma}_{\mathbf{1 2}} \\
(1 / \mathrm{cm})\end{array}$ \\
\hline \multirow[t]{2}{*}{1} & 1 & 1.4360 & 0.0095042 & 0.0058708 & \multirow[t]{2}{*}{0.017754} \\
\hline & 2 & 0.3635 & 0.0750580 & 0.0960670 & \\
\hline \multirow[t]{2}{*}{2} & 1 & 1.4366 & 0.0096785 & 0.0061908 & \multirow[t]{2}{*}{0.017621} \\
\hline & 2 & 0.3636 & 0.0784360 & 0.1035800 & \\
\hline \multirow[t]{2}{*}{3} & 1 & 1.3200 & 0.0026562 & 0.0000000 & \multirow[t]{2}{*}{0.023106} \\
\hline & 2 & 0.2772 & 0.0715960 & 0.0000000 & \\
\hline \multirow[t]{2}{*}{4} & 1 & 1.4389 & 0.0103630 & 0.0074527 & \multirow[t]{2}{*}{0.017101} \\
\hline & 2 & 0.3638 & 0.0914080 & 0.1323600 & \\
\hline \multirow[t]{2}{*}{5} & 1 & 1.4381 & 0.0100030 & 0.0061908 & \multirow[t]{2}{*}{0.017290} \\
\hline & 2 & 0.3665 & 0.0848280 & 0.1035800 & \\
\hline \multirow[t]{2}{*}{6} & 1 & 1.4385 & 0.0101320 & 0.0064285 & \multirow[t]{2}{*}{0.017192} \\
\hline & 2 & 0.3665 & 0.0873140 & 0.1091100 & \\
\hline \multirow[t]{2}{*}{7} & 1 & 1.4389 & 0.0101650 & 0.0061908 & \multirow[t]{2}{*}{0.017125} \\
\hline & 2 & 0.3679 & 0.0880240 & 0.1035800 & \\
\hline \multirow[t]{2}{*}{8} & 1 & 1.4393 & 0.0102940 & 0.0064285 & \multirow[t]{2}{*}{0.017027} \\
\hline & 2 & 0.3680 & 0.0905100 & 0.1091100 & \\
\hline Material & $\mathrm{g}$ & $\delta D_{g}$ & $\delta \Sigma_{a g}$ & $\nu \delta \Sigma_{f g}$ & $\delta \Sigma_{12}$ \\
\hline \multirow{2}{*}{$\begin{array}{c}\text { Perturbed } \\
\text { Region I }\end{array}$} & 1 & 0.0000000 & $-0.0009668 \mathrm{i}$ & $-0.0003642 \mathrm{i}$ & \multirow[t]{2}{*}{$+0.0009330 \mathrm{i}$} \\
\hline & 2 & 0.0000000 & $-0.0190000 \mathrm{i}$ & $-0.0085000 \mathrm{i}$ & \\
\hline \multirow{2}{*}{$\begin{array}{c}\text { Perturbed } \\
\text { Region II }\end{array}$} & 1 & 0.0000000 & $+0.0009668 \mathrm{i}$ & $+0.0003642 \mathrm{i}$ & \multirow[t]{2}{*}{$-0.0009330 \mathrm{i}$} \\
\hline & 2 & 0.0000000 & $+0.0190000 \mathrm{i}$ & $+0.0085000 \mathrm{i}$ & \\
\hline \multirow{2}{*}{$\begin{array}{c}\text { Non Perturbed } \\
\text { Region }\end{array}$} & 1 & 0.0000000 & 0.0000000 & 0.0000000 & \multirow[t]{2}{*}{0.000000} \\
\hline & 2 & 0.0000000 & 0.0000000 & 0.0000000 & \\
\hline
\end{tabular}

change along the FA movement.

When, any kind of a perturbation in a critical system without thermal-hydraulic feedback is introduced — even a sinusoidal perturbation - the system becomes unstable, since $k_{\text {eff }}$ deviates from unity. Nevertheless, this change in criticality will be attenuated by the thermal-hydraulic feedback that has a stabilizing effect. As Figure 6 shows that maximum static reactivity is only $\rho=\left(k_{\text {eff }}-1\right) / k_{\text {eff }} \simeq 1.5 \times 10^{-8}$. Even if it is possible to introduce some kind of average kinetic eigenvalue to mitigate this effect [31]. This correction was not implemented in this work because of the smallness of the perturbation in reactivity. If the simulation was longer or the perturbation was greater, this kind of treatment would be necessary.

Figure 7 shows a comparison of the relative amplitude of neutron flux noise for the fast and thermal groups along $y=150.2969 \mathrm{~cm}$ obtained with the FEMFFUSION code and the CORE SIM code, in other words, between the time-domain and the frequency-domain approaches. Figure 8 gives a 


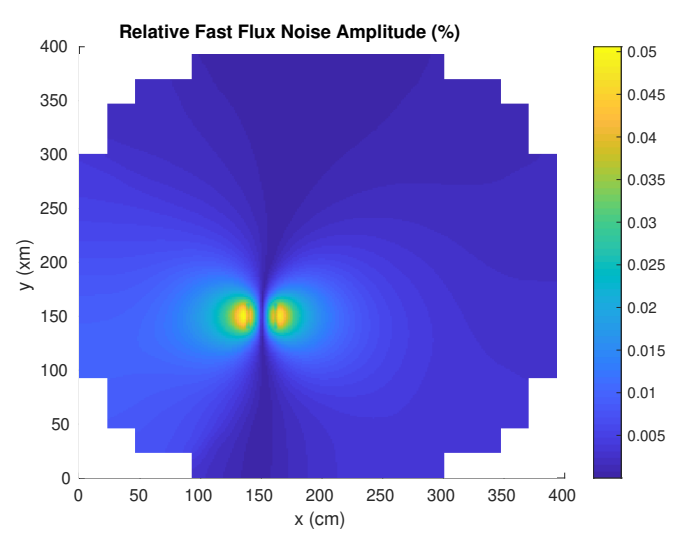

(a) Fast flux noise

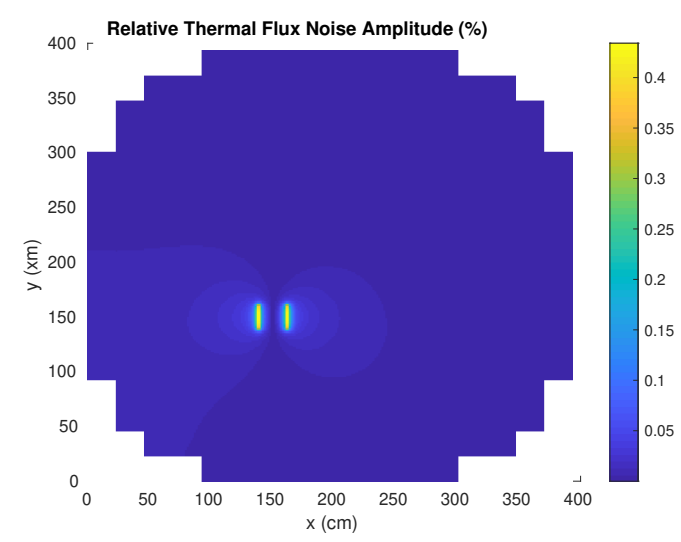

(b) Thermal flux noise

Fig. 5. Noise amplitudes for the 2D BIBLIS reactor at $1 \mathrm{~Hz}$.

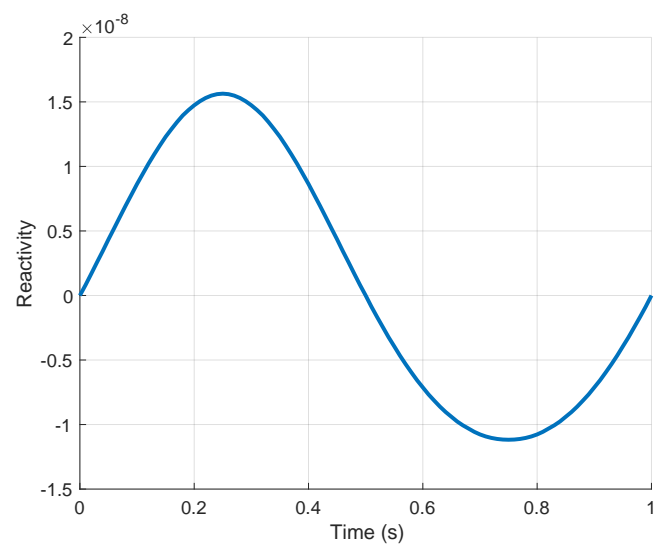

Fig. 6. Variation of the reactivity for the 2D BIBLIS reactor.

comparison of the phase of the neutron noise obtained with the two codes. A close agreement is observed for both the amplitude and the phase of the neutron noise for the vibration frequency at $1 \mathrm{~Hz}$, especially far from the FA vibration. We can observe that the vibrating FA produces a phase change from $90^{\circ}$ to $-90^{\circ}$ in the induced noise.

Figure 9 shows the relative error in the noise amplitude different along the line $y=150.2969$ $\mathrm{cm}$ when different time steps are used. This Figure demonstrates that a time step of $10^{-2} \mathrm{~s}$ is enough to accurately reproduce the noise amplitude results.

The relative difference between these approaches is quantified in Figure 10. The relative differences are small in regions far away from the perturbation, although the relative differences at the perturbation location are comparably bigger. This difference is mainly caused by the spatial 
discretization error in the time-domain and the frequency-domain methodologies. The frequencydomain approach is based on a finite difference approach, whereas the time-domain approach is based on a finite element approach. The latter approach is known to better capture steep gradients, as is the case close to the noise source.

Figure 11 displays the amplitude for the second harmonic of the neutron, at $2 \mathrm{~Hz}$, calculated with the time-dependent code. It can be observed that this noise is sharper than the noise associated with the fundamental frequency and its amplitude much smaller than the $1 \mathrm{~Hz}$ component. In this Figure, it can be seen that there are two drops of noise amplitude in the middle of the moving interfaces as the ones shown in the frequency analysis of the cross section perturbation in Figure 3(b). Figure 12 shows the amplitude of the neutron noise for the third harmonic at 3 Hz. These higher harmonics have less influence on the total neutron noise because their amplitude are much smaller than the fundamental harmonic of the noise at the FA vibration frequency. The contributions of the multiples frequencies come from the fact that the perturbation source has different frequencies. In the time-domain simulations moreover, the multiple frequencies are also due to the explicit modeling of the non-linearities. However, the harmonics following the first one are much smaller, and cancel out in adjacent regions. This causes that these harmonics have a very small noise amplitude. Thus, the neutron noise at the FA frequency is the main component of the neutron noise and must be the one used to detect anomalies in the operation of a nuclear reactor.

It should also be pointed out that the analysis of plant data is typically performed using frequency spectra of neutron detectors, i.e. the effect of vibrations is thus resolved at different frequencies. If only the fundamental frequency is considered, the present work demonstrates that estimating the effect of a FA vibration can be equally well carried out using modeling in the time domain or in the frequency-domain (using a first order approximation).

\section{CONCLUSIONS}

This research presented the modeling of the effect of the mechanical vibration of fuel assemblies onto neutron noise. The problem combines different spatial scales, e.g. a $1 \mathrm{~mm}$ oscillation of a fuel assembly that measures about $20 \mathrm{~cm}$ in radial size in a reactor of some meters length. This implies that we need to work with a very high precision in the spatial discretization and in the 
tolerances given to the solvers.

Numerical results show that the main oscillation in the neutron flux is obtained at the same frequency as the one of the assembly vibration. Neutron noise at multiples of the mechanical vibration are also seen in time-dependent calculation but with much less amplitude. This paper compares the time-domain analysis and the frequency-domain analysis of the phenomena. Numerical results show a close match between these two approaches at the FA vibration frequency.

Higher harmonics are less important to total neutron noise because their amplitude is much smaller than the fundamental harmonic. Furthermore, the neutron noise corresponding to those higher harmonics has a much larger spatial decay away from the perturbation. It thus means that unfolding the location of the vibrating FA from distant neutron detectors is far more challenging than using the neutron noise at the fundamental frequency.

\section{ACKNOWLEDGMENTS}

This project has received funding from the Euratom research and training programme 20142018 under grant agreement No 754316. Also, this work has been partially supported by Spanish Ministerio de Economía y Competitividad under project BES-2015-072901 and financed with the help of Primeros Proyectos de Investigación (PAID-06-18), Vicerrectorado de Investigación, Innovación y Transferencia of the Universitat Politècnica de València (UPV).

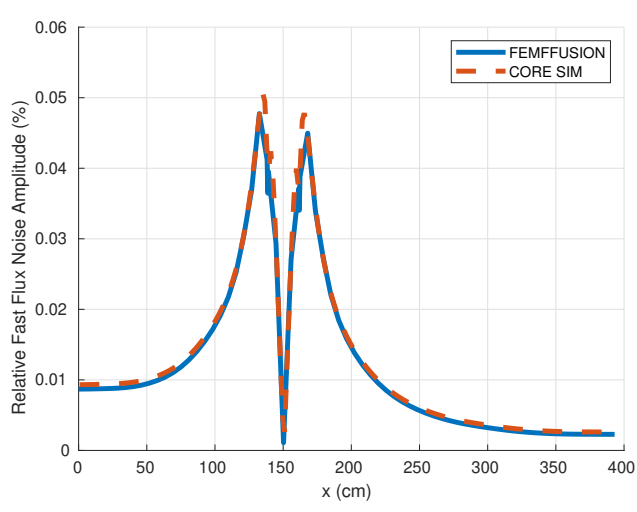

(a) Fast flux noise

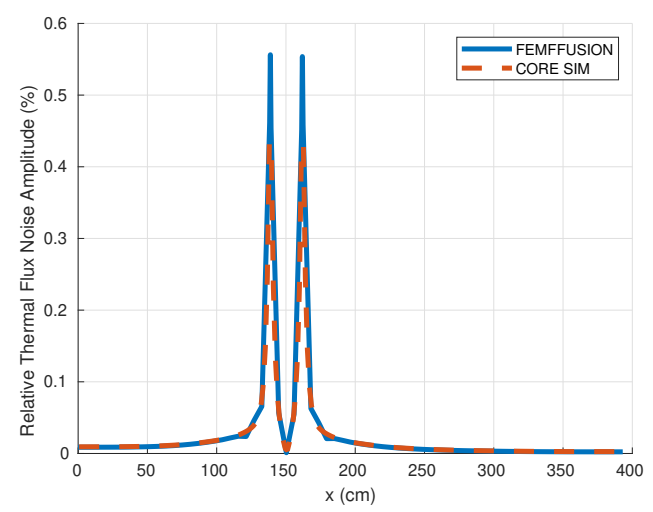

(b) Thermal flux noise

Fig. 7. Relative noise amplitude comparison for $2 \mathrm{D}$ BIBLIS reactor in $\mathbf{y}=\mathbf{1 5 0 . 2 9 6 9} \mathrm{cm}$ at $1 \mathrm{~Hz}$. 


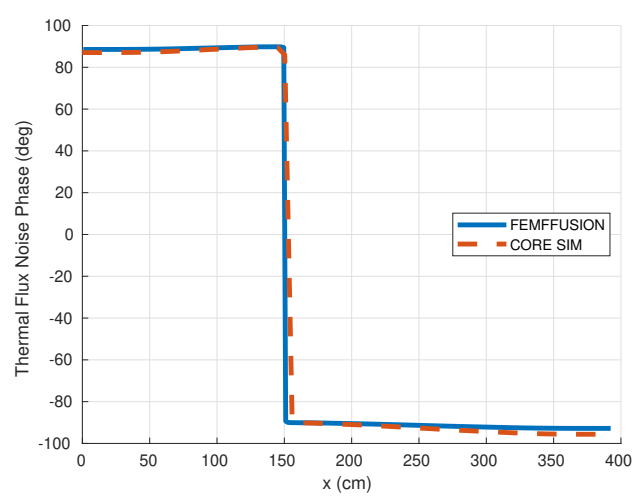

(a) Fast flux noise

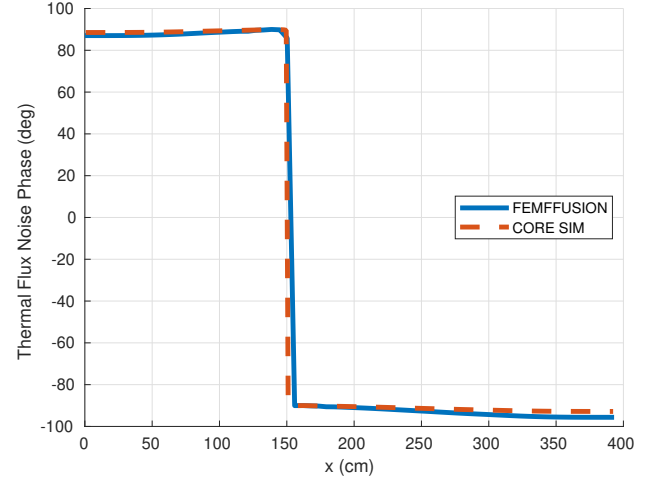

(b) Thermal flux noise

Fig. 8. Phase comparison for 2D BIBLIS reactor in $y=150.2969 \mathrm{~cm}$ at $1 \mathrm{~Hz}$.

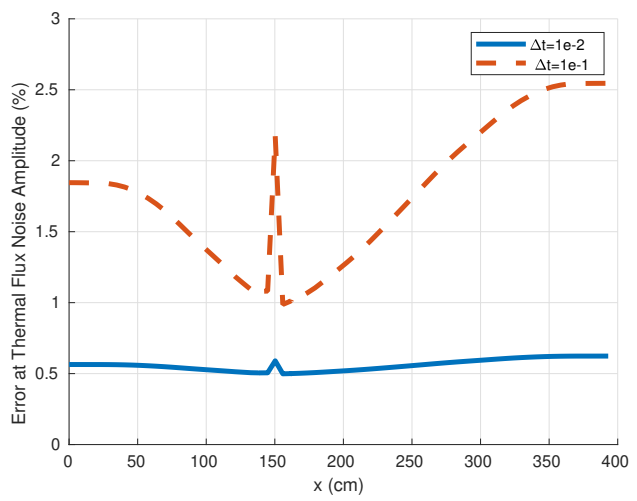

(a) Fast flux noise

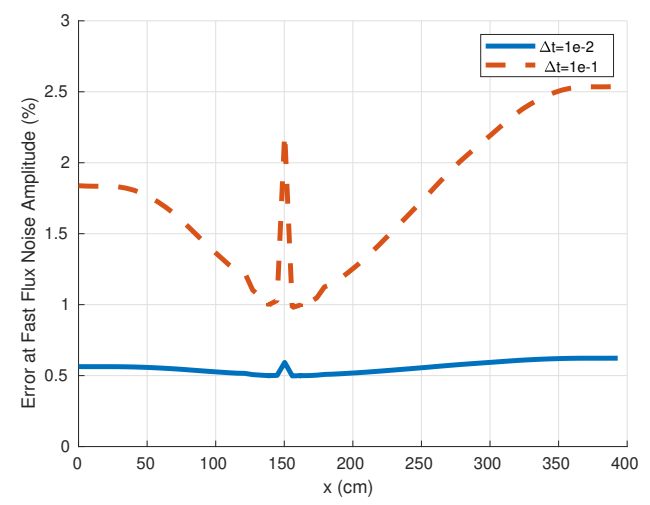

(b) Thermal flux noise

Fig. 9. Relative error in noise amplitude at different time steps for 2D BIBLIS reactor in $\mathbf{y}=\mathbf{1 5 0 . 2 9 6 9} \mathrm{cm}$ at $1 \mathrm{~Hz}$.

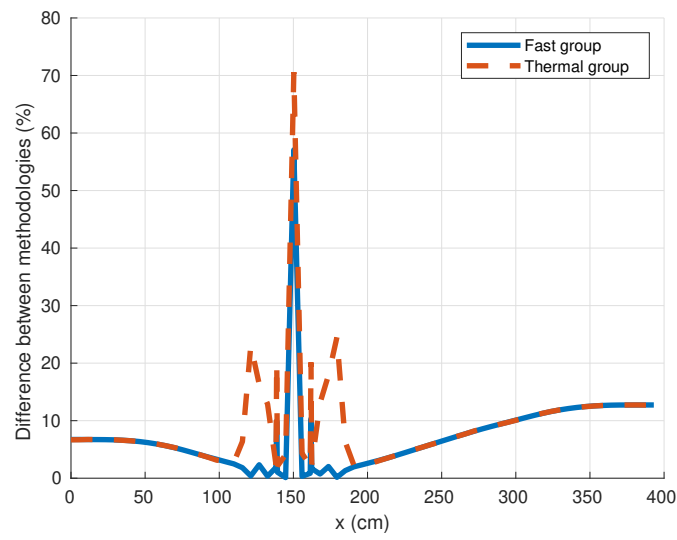

Fig. 10. Relative difference in noise amplitude between frequency-domain and time-domain methodologies for 2D BIBLIS reactor in $\mathbf{y}=\mathbf{1 5 0 . 2 9 6 9} \mathrm{cm}$ at $1 \mathrm{~Hz}$. 


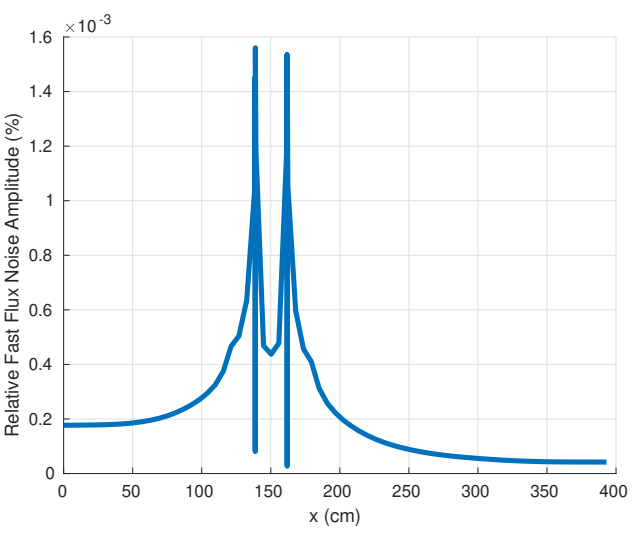

(a) Fast flux noise

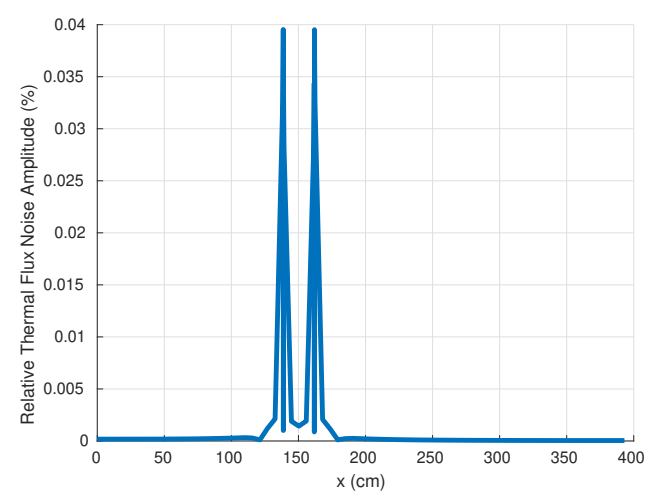

(b) Thermal flux noise.

Fig. 11. Relative noise amplitudes in $y=150.2969 \mathrm{~cm}$ at $2 \mathrm{~Hz}$.

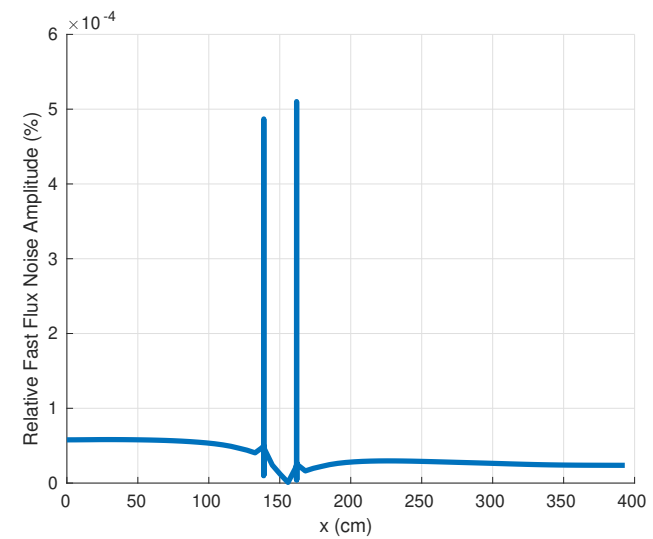

(a) Fast flux noise

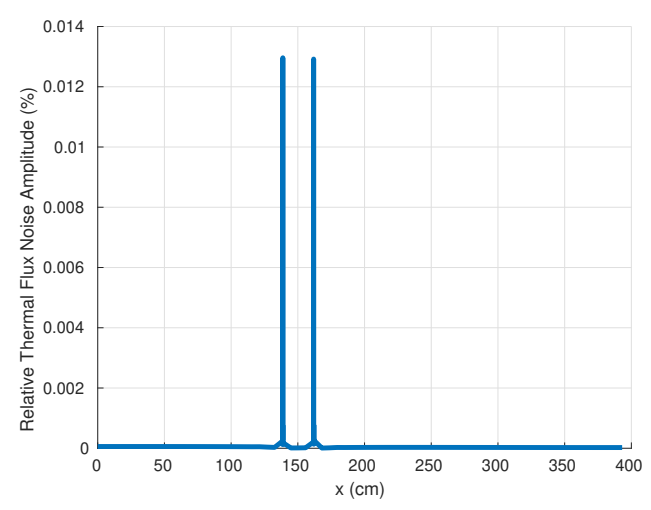

(b) Thermal flux noise

Fig. 12. Relative noise amplitudes in $y=150.2969 \mathrm{~cm}$ at $3 \mathrm{~Hz}$. 


\section{REFERENCES}

[1] C. Demazière, P. Vinai, M. Hursin, S. Kollias, and J. Herb, "Noise-Based Core Monitoring and Diagnostics - Overview of the project," Advances in Reactor Physics (ARP-2017), 1-4, Mumbai, India (2017).

[2] M. Seidl, K. Kosowski, U. Schüler, and L. Belblidia, "Review of the historic neutron noise behavior in German KWU built PWRs," Prog. Nucl. Energy, 85, 668 (2015); 10.1016/j.pnucene.2015.08.016.

[3] D. Fry, J. March-Leuba, and F. Sweeney, "Use of neutron noise for diagnosis of in-vessel anomalies in light-water reactors," NUREG/CR-3303, Oak Ridge National Lab., TN (USA) (1984).

[4] D. N. FrY, "Experience in Reactor Malfunction Diagnosis Using On-Line Noise Analysis," Nuclear Technology, 10, 3, 273 (1971); 10.13182/nt71-a30959.

[5] V. Lamirand, O. Pakari, and A. Laureau, "Experimental report of the 1st campaign at AKR-2 and CROCUS," CORTEX deliverable D2.1, EPFL (2018)URL https://ec.europa.eu/research/participants/documents/downloadPublic? document Ids $=080166 \mathrm{e} 5 \mathrm{bf}$ af 5 ce8\&appId=PPGMS.

[6] D. Chionis, A. Dokhane, L. Belblidia, M. Pecchia, G. Girardin, H. Ferroukhi, and A. PAutz, "SIMUlATE-3K Analyses of Neutron Noise Response to Fuel Assembly Vibrations and Thermal-Hydraulics Parameters Fluctuations," MEGC 2017, 1-8 (2017).

[7] M. Viebach, S. Kliem, C. Demazière, U. Rohde, D. Henning, and H. A., "A Comparison between time domain and frecuency domain calculations of stationary neutron fluctuations," Proceedings of the International Conference on Mathematics and Computational Methods Applied to Nuclear Science and Engineering (MESC 2019). Portland, Oregon, USA, August 25-29, 2019, on CD-ROM, American Nuclear Society (ANS) (2019).

[8] N. Olmo-Juan, C. Demazière, T. Barrachina, R. Miró, and G. Verdú, "Parcs vs CORE SIM neutron noise simulations," Prog. Nucl. Energy, 115, 169 (2019); 10.1016/j.pnucene.2019.03.041. 
[9] A. Vidal-Ferràndiz, A. Carreño, D. Ginestar, C. Demazière, and G. Verdú, "A time and frequency domain analysis of the effect of vibrating fuel assemblies on the neutron noise," Ann. Nucl. Energy, 137, 107076 (2020); 10.1016/j.anucene.2019.107076.

[10] J. Park, J. H. Lee, T.-R. Kim, J.-B. Park, S. K. Lee, and I.-S. Koo, "Identification of reactor internals' vibration modes of a Korean standard PWR using structural modeling and neutron noise analysis," Prog. Nucl. Energy, 43, 1-4, 177 (2003); 10.1016/S01491970(03)00021-0.

[11] A. Vidal-Ferràndiz, A. Carreño, D. Ginestar, C. Demazière, and G. Verdú, "Neutronic Simulation of Fuel Assembly Vibrations in a Nuclear Reactor," Int. Conf. Mathematics ES Computational Methods Applied to Nuclear Science \& Engineering (MEC 2019), 1-8 (2019).

[12] W. M. Stacey, Nuclear Reactor Physics, Wiley, Weinheim, Germany (2007); $10.1002 / 9783527611041$.

[13] T. Yамамото, "Monte Carlo method with complex weights for neutron leakage-corrected calculations and anisotropic diffusion coefficient generations," Annals of Nuclear Energy, 50, 141 (2012); 10.1016/j.anucene.2012.06.025.

[14] A. Mylonakis, H. Yi, P. Vinai, and C. Demazière, "Neutron noise simulations in a heterogeneous system: A comparison between a diffusion-based and a discrete ordinates solver," Proc. Int. Conf. Mathematics $\&$ Computational Methods Applied to Nuclear Science $\&$ Engineering (MEC 2019) (2019).

[15] A. Vidal-Ferràndiz, R. Fayez, D. Ginestar, and G. Verdú, "Solution of the Lambda modes problem of a nuclear power reactor using an hp finite element method," Ann. Nucl. Energy, 72, 0, 338 (2014).

[16] A. Vidal-Ferràndiz, R. Fayez, D. Ginestar, and G. Verdú, "Moving meshes to solve the time-dependent neutron diffusion equation in hexagonal geometry," J. Comput. Appl. Math., 291, 197 (2016); 10.1016/j.cam.2015.03.040.

[17] D. Ginestar, G. Verdú, V. Vidal, R. Bru, J. Marín, and J. Muñoz-Cobo, "High order backward discretization of the neutron diffusion equation," Ann. Nucl. Energy, 25, 1-3, 47 (1998); 10.1016/s0306-4549(97)00046-7. 
[18] Y. SAAD, Iterative Methods for Sparse Linear Systems, Society for Industrial and Applied Mathematics, Philadelphia, PA, USA (2003).

[19] W. Bangerth, R. Hartmann, and G. Kanschat, "deal.II - a General Purpose Object Oriented Finite Element Library," ACM Trans. Math. Softw., 33, 4, 24/1 (2007).

[20] S. Balay, S. Abhyankar, M. F. Adams, J. Brown, P. Brune, K. Buschelman, L. Dalcin, A. Dener, V. Eijkhout, W. D. Gropp, D. Karpeyev, D. Kaushik, M. G. KnePley, D. A. May, L. C. McInnes, R. T. Mills, T. Munson, K. Rupp, P. Sanan, B. F. Smith, S. Zampini, H. Zhang, and H. Zhang, "PETSc Users Manual," ANL-95/11 Revision 3.12, Argonne National Laboratory (2019)URL https://www.mcs.anl.gov/petsc.

[21] V. Hernandez, J. E. Roman, and V. Vidal, "SlEPc: A Scalable and Flexible Toolkit for the Solution of Eigenvalue Problems," ACM Trans. Math. Software, 31, 3, 351 (2005).

[22] C. Demazière and G. Andhill, "Identification and localization of absorbers of variable strength in nuclear reactors," Ann. Nucl. Energy, 32, 8, 812 (2005); 10.1016/j.anucene.2004.12.011.

[23] C. Demazière, "Analysis methods for the determination of possible unseated fuel assemblies in BWRs," Int. J. Nucl. Ener. Sci. Tech., 2, 3, 167 (2006); 10.1504/ijnest.2006.010713.

[24] C. Demazière and I. PÁzsit, "Numerical tools applied to power reactor noise analysis," Prog. Nucl. Energy, 51, 1, 67 (2009); 10.1016/j.pnucene.2008.01.010.

[25] C. Demazière, "CORE SIM: A multi-purpose neutronic tool for research and education," Ann. Nucl. Energy, 38, 12, 2698 (2011); 10.1016/j.anucene.2011.06.010.

[26] C. Demazière, "Validation and demonstration of the CORE SIM neutronic tool," CTH-NT242, Chalmers University of Technology (2011).

[27] I. PÁzsit and C. Demazière, "Noise Techniques in Nuclear Systems," Handbook of Nuclear Engineering, 1629-1737, Springer US.

[28] A. Rouchon and R. Sanchez, "Analysis of vibration-induced neutron noise using onedimension noise diffusion theory," ICAPP 2015 - International Congress on Advances in Nuclear Power Plants, Nice, France (2015). 
[29] A. Rouchon, "Analysis and development of deterministic and stochastic neutron noise computing techniques with applications to thermal and fast reactors," $\mathrm{PhD}$ Thesis, Université Paris-Saclay (2016)URL https://tel.archives-ouvertes.fr/tel-01381245.

[30] A. HÉBERT, "Application of the Hermite Method for Finite Element Reactor Calculations," Nucl. Sci. Eng., 91, 1, 34 (1985); 10.13182/nse85-a17127.

[31] A. Gammicchia, S. Santandrea, I. Zmijarevic, R. Sanchez, Z. Stankovski, S. Dulla, and P. Mosca, "A MOC-based neutron kinetics model for noise analysis," Annals of Nuclear Energy, 137, 107070 (2020); 10.1016/j.anucene.2019.107070. 